\title{
Customized Orthodontic Mini-screw Surgical Template Design and Manufacture
}

\author{
Chun-Li Lina, ${ }^{\text {a, }}$ Wen-Jen Chang ${ }^{\mathrm{b}}$, Yu-Tzu Wang a , Pin-Hsin Hsu ${ }^{\mathrm{a}}$ \\ a Department of Biomedical Engineering, National Yang-Ming University, 112 Taipei, Taiwan \\ ${ }^{b}$ Department of Information Management, Chang Gung University, 333 Tao-yuan, Taiwan. \\ *Corresponding Author: cllin2@ym.edu.tw
}

\begin{abstract}
The aim of this study was to develop a technique which integrated cone-beam computed tomography (CBCT)/laser scan image superposition, computer-aided design (CAD) and rapid prototyping (RP) for producing customized orthodontic mini-screw surgical templates. Maxillary bone and teeth images were obtained by CBCT and mucosa outer profile was acquired by digital laser scan. CT and laser scanned images were superimposed to allow mini-screw visual insertion planning and permit surgical template fabrication. The customized surgical template CAD model was fabricated offset based on the teeth/mucosa/bracket contour profiles in the superimposition model and exported to duplicate the plastic template using the rapid prototyping technique. A clinical test for the maxillary third molar showed that mini-screw was placed safely and did not produce inflammation or other discomfort symptoms one week after surgery. The fitness between the mucosa and template indicated that the average gap sizes were found smaller than $0.5 \mathrm{~mm}$ and confirmed that the surgical template presented good holding power and well-fitting adaption. This study addressed integrating CBCT and laser scan image superposition, CAD and RP techniques can be applied to fabricate an accurate customized surgical template for orthodontic mini-screws.
\end{abstract}

Keywords: CBCT, RP, mini-screw, orthodontic.

\section{Introduction}

The orthodontic mini-screw provides skeletal anchorage with the advantages of relatively inexpensive, easily implemented and predictable enough to be used routinely in medical practice [1]. Nonetheless, the failure rates of interradiculary inserted mini-screws are considered still too high [2]. The mini-screw placement poses a challenge to the orthodontist because of the limited space available for miniscrew placement and the potential risk for root damage, puncture to the maxillary sinus and neurovascular damage during mini-screw placement procedures [3]. A controllable method for mini-screw placement and direction is important for orthodontists.

Two-dimensional information in the radiographs is usually used for surgical sites planning to minimize the root damage risks [4-6]. Custom-made surgical guides and templates have recently been proposed for transferring computed tomography (CT) images to the surgical site and outlining the ideal mini-screw axis, to promote safe miniscrew placement into the dentoalveolar bone [7,8]. However, fabricating the accuracy and fit of individualized surgical guides is time-consuming and requires extensive advance preparation because CT images only isolate the hard tissue positions (jawbone or teeth). Complex double CT scan, landmark definition and image processing procedures are needed to identify the accurate position and thickness of the soft tissue (mucosa) that comes in direct contact with the surgical templates $[1,4]$.

This study integrates image superposition of $\mathrm{CBCT} /$ laser scanning, computer aided design (CAD) and rapid prototyping technologies to develop a simplified, accurate technique for producing customized mini-screw surgical templates with adaptive fitness between the mucosa and template for accurate mini-screw insertion paths.

\section{Materials and Methods}

\subsection{Image superimposition and mini-screw inserted path definition}

The patient was planned to insert a dual-thread miniscrew (materials: Ti6Al4V, Bomei Co, Ltd, Taoyuan, Taiwan) $1.6 \mathrm{~mm}$ in diameter and $8 \mathrm{~mm}$ in length as the anchor implant at the molar space of the left buccal site (Figs. 1 (a) 
and (b)).

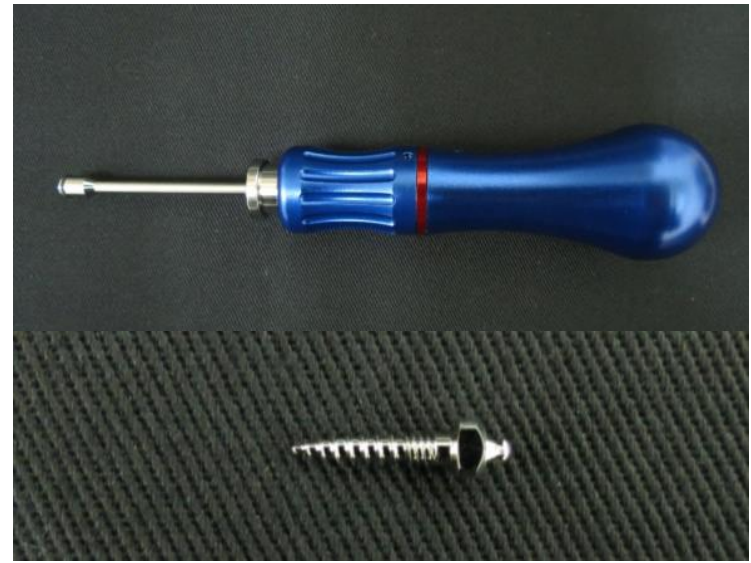

(a)

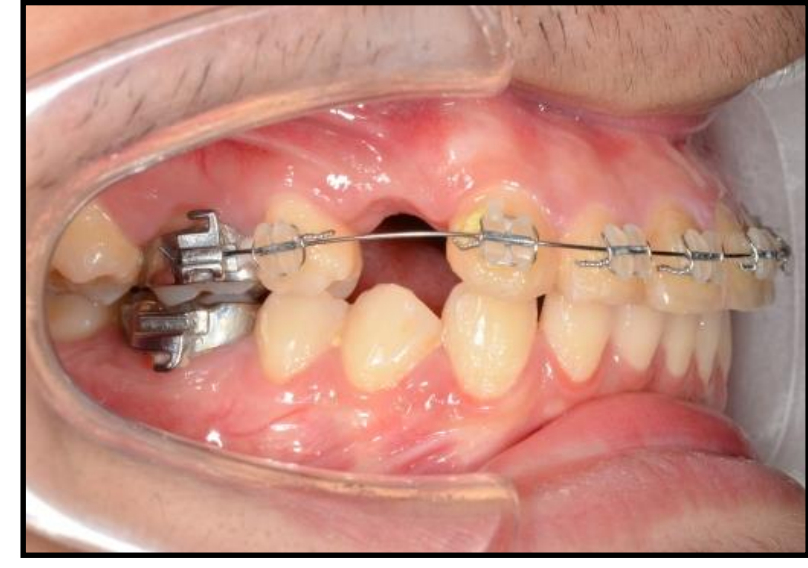

(b)

Fig. 1 (a) Dual-thread mini-screw 1.6mm in diameter and $8 \mathrm{~mm}$ in length was selected as the anchor implants; (b) mini-screw was planned for insertion at the molar space of the right buccal sites.

CBCT (Cone-Beam Computed Tomography, Asahi AZ3000, Kyoto-shi, Japan) scan was performed on the patient to reconstruct the 3D maxillae model. (Figs. 2 (a) and (b)). In order to ensure that the surgical template would fit well with the patient's teeth and soft tissue (mucosa), a maxilla impression was taken to make a stone cast. The stone cast was then scanned using a 3D surface laser scan system (3Shape Scanners, 3D Scan CO., Ltd., USA) to make a digital 3D impression model for recording the geometry and profile of the teeth and mucosa. Solid models of maxillary

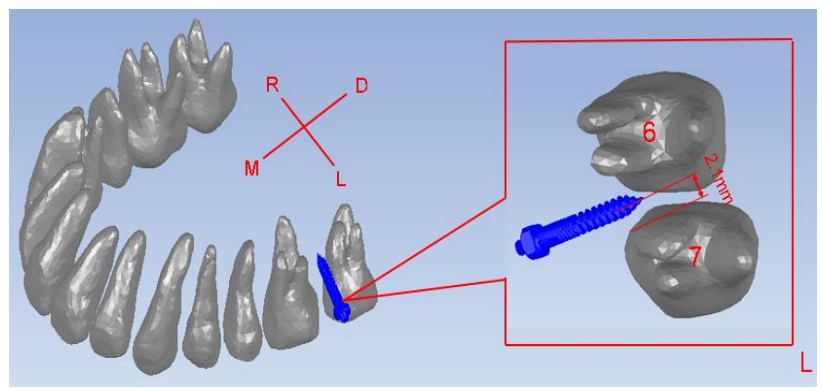

(a) bone with teeth reconstructed using $\mathrm{CBCT}$ images and maxillary dental arches with mucosa acquired using laser scanning were superimposed using the common register positions at the disto-inciso angle of left/right incisors and disto-buccal cusp of left/right first molar. The superimposition model consisted of hard tissue (bone and teeth) positions to allow mini-screw visual insertion planning accurate orientation and teeth/mucosa/bracket contour profiles to permit surgical template fabrication with wellfitting adaption.

(b)

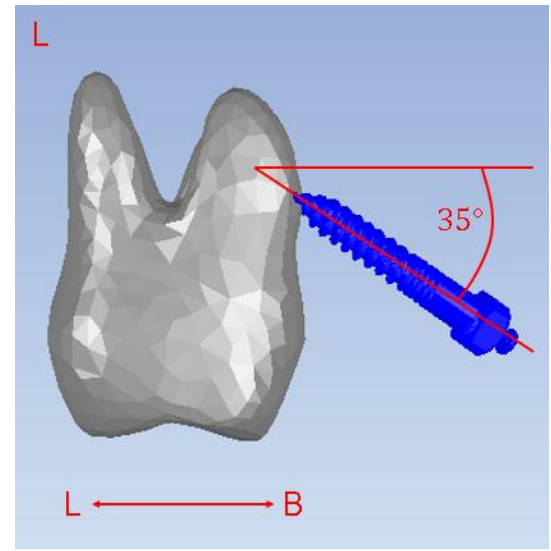

Fig. 2 (a) 3D solid model of the maxillary bone with teeth was reconstructed for surgical mini-screw planning at left site; (b) mini-screw was planned to insert to the occlusal surface inclination at angles of $35^{\circ}$.

The CAD mini-screw models were imported and placed in safe and optimal positions (Figs. 2 (a)). The distance between the first and second molars roots for the mini-screw was measured. The periodontal ligament is about 0.15 to 0.38 $\mathrm{mm}$ thick on average (assumed as $0.4 \mathrm{~mm}$ in this study) and the mini-screw diameter in this study was $1.6 \mathrm{~mm}$. The safe distance between the second premolar root and mini-screw axis was calculated and should be larger than $1.2 \mathrm{~mm}$, i.e. the 
sum of the periodontal ligament thickness $0.4 \mathrm{~mm}$ and miniscrew radius $0.8 \mathrm{~mm}$ for safe placement. Mini-screw was inserted to the occlusal surface inclination at angles of $35^{\circ}$ for the left placement site (Fig. 2(b)).

\subsection{Surgical template fabrication and clinical test}

The customized surgical template CAD model was fabricated with a 1.8-mm-thick layer average offset based on the teeth/mucosa/bracket contour profiles in previous superimposition models, ranging from half canine to second molar (Fig. 3). The surgical template height was designed to

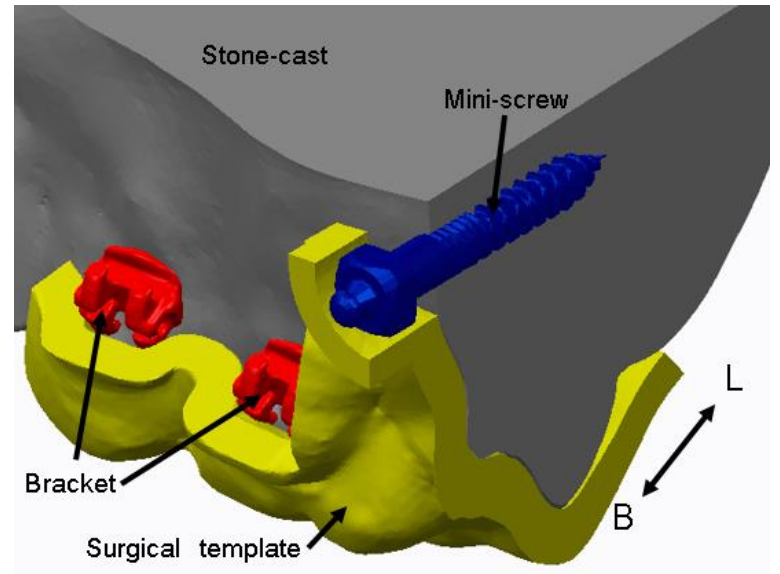

Fig. 3 The customized surgical template.

The surgical templates were placed intra-orally onto the teeth by a clinician. Once the template was seated the patient was asked to bite the template to expose the guide surgical sites. The mini-screws were then placed with a screwdriver (Fig. 5) until the screw head bottom slightly touched the mucosa. X-rays were taken after implantation to assess whether damage to the peripheral tissue occurred for assessing mini-screw stability.

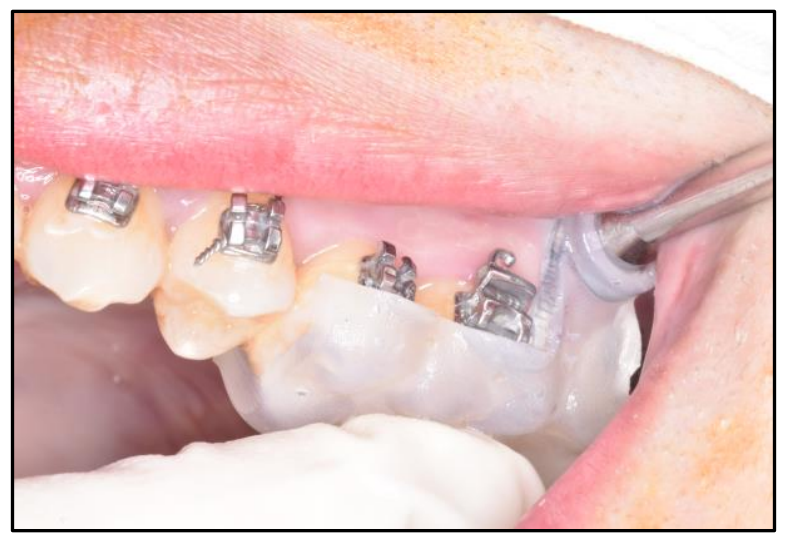

Fig. 5 Clinical application of the customized surgical template. cover the entire occlusal surface and extend to half the crown height to protect the orthodontic brackets bonded onto each tooth. The mini-screw drill paths and guided cylinders (5.3 $\mathrm{mm}$ in diameter and $6 \mathrm{~mm}$ in height) were generated in the surgical template CAD model according to previous 3D information from the visual surgical plan (Fig. 3). The solid surgical template model can be exported as a stereolithographic (STL) file that can be loaded into a fused deposition modeling (FDM) rapid prototyping (RP) printer (Dimension 1200es SST, Strayasys, Ltd., Minnesota, USA) to duplicate the ABS (ABS-P430, Strayasys, Ltd., Minnesota, USA) plastic template (Fig. 4).

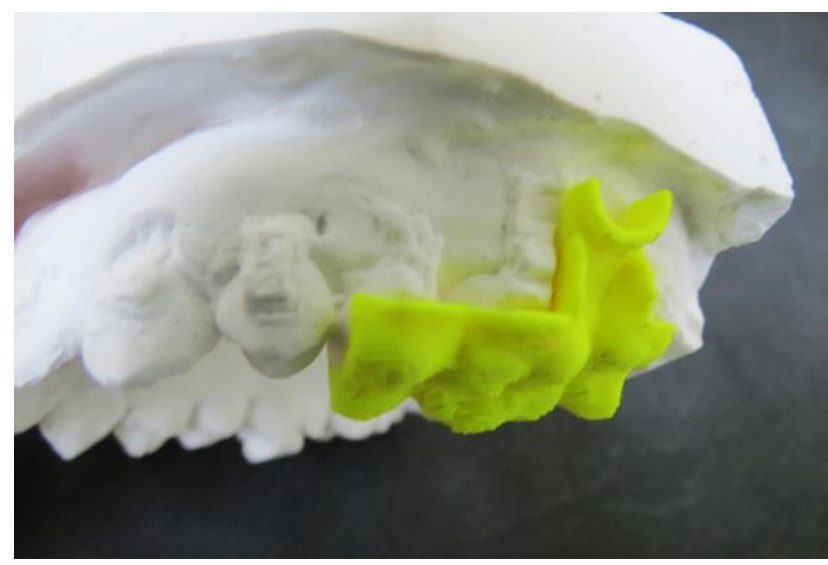

Fig. 4 The RP surgical template (left site) wore on the stone cast.

\section{Results and Discussion}

The clinical application indicated that two mini-screws were placed smoothly, safety and without problems. No inflammation, screw loosening or other symptoms of discomfort had occurred one week after surgery.

Mini-screws are always placed at an inclined angle, obviously limiting 2D image measurement. CT scan therefore must be used to measure the interradicular spaces for accurate and reproducible results. However, the CT characteristic only presents/identifies deeper anatomical hard tissue, which often limits its applicability in surgery guiding templates. Much effort and numerous methods such as double CT scans for hard tissue and the radiographic template and landmark definition used with metal wires/balls were proposed to identify the contour profile and thickness of soft tissue (mucosa) that comes into direct contact with surgical templates $[1,4,7]$. This study proposes an image superimposition method using laser scan and CBCT images to improve the surgical template interfacial fitting adaptation 
problem. Laser scanning can be used as a complementary tool for designing surgical templates because the images obtained from laser scanning can present the patient's dental arch with a good degree of similarity and may enhance the holding power and stability of the surgical template.

The stability and inherent support of the surgical template is a crucial factor. The template in this study was supported using 3 surfaces - occusal teeth and buccal/palatal mucosa. The bite forces on the template provided support to keep it stable. In order to evaluate the interfacial fitness accuracy, an interfacial adaption test was performed to measure the gap sizes between the surgical template and teeth/mucosa tissue [1]. The customized surgical template was fitted onto teeth/mucosa stone cast models and embedded into clear rectangular test boxes with epoxy resin (Truetime Industrial Co., Taiwan) to provide a stable base. The resin block was sectioned in the buccal-palatal direction from the second premolar to molar with 6 section slices using a low speed diamond saw with copious cooling (CL50 Precision Saw, Top Tech Machines Co., Ltd., Taiwan). The section slices were scanned using a non-contact video measurement system (SVP-2010, ARCS Co., Ltd., Taichung, Taiwan) to measure the gap sizes at 5 points with $45^{\circ}$ separation in the counterclockwise direction on each section. The results showed that the average gap sizes in the different tooth sections were found to be smaller than $0.5 \mathrm{~mm}$ (total average $0.30 \pm 0.10 \mathrm{~mm}$ ) and confirmed that the image superimposed method can produce a surgical template with good holding power and well-fitting adaption.

The 3D superposition digital model from CBCT and laser scanning images produced accurate hard tissue relationship positions (bone and teeth) to simulate mini-screws placed in the safe position with ideal inclined angle and positions. Dual-thread mini-screws with correct micro thread pitch (parametrical relationship with macro thread pitch) in the cortical bone region can improve primary stability and enhance mechanical retention. This case indicated that the mini-screw micro thread can contact the cortical bone layer when the mini-screw head bottom is controlled to slightly touch the mucosa during insertion.

\section{Conclusions}

A new method for integrating CBCT/laser scan image superimposition, CAD system and RP techniques was developed in this study and applied to fabricate an accurate customized surgical template for orthodontic mini-screws. More clinical applications can be applied to verify the feasibility of the proposed method.

\section{References}

(1) Junji Sugawara: "Temporary skeletal anchorage devices: the case for miniscrews", American Journal of Orthodontics and Dentofacial Orthopedics, Vol. 145, pp. 558-564, 2014

(2) M Schätzle, R Männchen, M Zwahlen, et al: "Survival and failure rates of orthodontic temporary anchorage devices: a systematic review", Clinical Oral Implants Research, Vol. 20, pp. 1351-1359, 2009

(3) ND Kravitz and B Kusnoto: "Risks and complications of orthodontic miniscrews", American Journal of Orthodontics and Dentofacial Orthopedics, Vol. 131, pp. 43-51, 2007

(4) EY Suzuki and B Suzuki: "Accuracy of miniscrew implant placement with a 3-dimensional surgical guide", Journal of Oral Maxillofacial Surgery, Vol. 66(6), pp.1245-1252, 2008

(5) HM Kyung, HS Park and SM Bae: "Development of orthodontic miniscrew implants for intraoral anchorage", Journal of Clinical Orthodontic, Vol. 6, pp. 321-328, 2003

(6) SH Kyung, JH Choi and YC Park: "Miniscrew anchorage used to protract lower second molars into first molar extraction sites", Journal of Clinical Orthodontic, Vol. 37, pp. 575-579, 2003

(7) N Kitai, Y Yasuda and K Takada: “A stent fabricated on a selectively colored stereolithographic model for placement of orthodontic mini-implants", International Journal of Adult Orthodontics and Orthognathic Surgery, Vol. 17(4), pp. 264-266, 2002

(8) W Martin, M Heffernan and J Ruskin: "Template fabrication for a midpalatal orthodontic implant: Technical note", International Journal of Oral Maxillofacial Implants, Vol. 17, pp.720-722, 2002 